\title{
Prävention von Cannabiskonsum und -missbrauch: Evidenzbasiert oder nur gut gemeint?
}

R. Soellner ${ }^{1}$
D. Kleiber

\author{
Prevention of Cannabis Use and Abuse: Evidence-Based or Only Well-Meaning
}

\section{Zusammenfassung}

In diesem Beitrag wird Cannabisprävention in Deutschland aus verschiedenen Perspektiven beleuchtet. Einem historischen Abriss der Entwicklung der Drogenprävention und deren Implikationen für die Prävention des Cannabiskonsums folgt eine $\mathrm{Zu}-$ sammenstellung metaanalytischer Befunde zur Wirksamkeit von Präventionsstrategien. Daran anschließend werden Präventionsziele dargestellt, wie sie aus gesundheitspolitischer Sicht im Hinblick auf Cannabis formuliert werden. Die so theoretisch und empirisch abgeleiteten Anforderungen an Präventionsmaßnahmen bzw. -projekte im Suchtbereich dienen als Grundlage, um vier aktuelle cannabisspezifische Präventionsprojekte hinsichtlich ihrer theoretischen oder empirischen Fundierung und ihrer expliziten und impliziten Präventionsziele zu analysieren. Metaanalytischen Studien zur Wirksamkeit von Präventionsmaßnahmen zufolge kann Suchtprävention durchaus effektiv sein, obwohl sie alles in allem bisher nur mäßige bis moderate Effekte aufweist. Spezifische Ansätze zur primären Prävention von Cannabis liegen allerdings bisher - auch international nicht vor. Alle Evidenz zur Wirksamkeit der Suchtprävention wurde bisher cannabisunspezifisch ermittelt. Eine einheitliche, schlüssige und in ihren Aussagen konsistente Präventionsbotschaft ist in Deutschland derzeit nur schwer erkennbar. Die Analyse der aktuellen cannabisspezifischen Präventionsprojekte zeigt, dass das deutsche Suchthilfesystem sich hauptsächlich an bereits riskant konsumierende Personen, Jugendliche und junge Erwachsene richtet. Die Subgruppen der noch nicht Suchtgefährdeten werden in den präventiven und interventiven Angeboten nicht explizit berücksichtigt. Als Ziel dieser Maßnahmen wurde

\section{Abstract}

In this paper prevention of cannabis will be highlighted from different perspectives. Following a historical review of the development of drug prevention in Germany and its implications for the prevention of cannabis use, results of meta analytic studies concerning the effectiveness of prevention programs will be presented. Prevention goals like they are formulated by health politicians are analysed then. On the basis of these empirical and theoretical deduced arguments four prevention projects dealing especially with cannabis will be analysed in relation to their theoretical and empirical foundation as well as their implicit and explicit goals. A review of meta-analytical studies shows that drug prevention might be successful, even if the effects are at best moderate. Cannabis specific primary prevention programs do not exist yet. Thus measures of evidence have been revealed in general drug prevention projects only. In Germany a holistic and consistent prevention message is not to be seen. It is shown that current cannabis specific prevention programs address predominantly adolescents and adults with problematic use behaviour. Subgroups of cannabis users with non-problematic use behaviour thus are not considered explicitly. Prevention goals are abstinence or reduction yielding in abstinence. The existence of a non problematic cannabis use is ignored. Finally the question of an adequate primary prevention goal is raised. Taken into account the evidence of such programs as well as results of developmental psychological oriented drug research it is argued that primary prevention referring to abstinence as the main prevention goal has serious shortcomings.

Institutsangaben

${ }^{1}$ Fachbereich 12, Arbeitsbereich Evaluation, Qualitätssicherung und Qualitätsmanagement in

Erziehungswissenschaft und Psychologie, Freie Universität Berlin

${ }^{2}$ Fachbereich 12, Arbeitsbereich Prävention und psychosoziale Gesundheitsforschung, Freie Universität Berlin

Korrespondenzadresse

Prof. Dr. Renate Soellner · FU-Berlin, FB 12, Arbeitsbereich Evaluation, Qualitätssicherung und

Qualitätsmanagement in Erziehungswissenschaft und Psychologie · Habelschwerdter Allee 45 • 14195 Berlin .

E-mail: soellner@zedat.fu-berlin.de

Bibliografie

Suchttherapie 2005; 6: 116-125 @ Georg Thieme Verlag KG Stuttgart · New York

DOI 10.1055/s-2005-858659

ISSN 1439-9903 
die Abstinenz oder die Reduktion mit anschließender Abstinenz festgestellt. Dass es einen nicht problematischen Konsum von Cannabis geben kann, wird ausgeklammert. Dies führt zu der Frage nach dem adäquaten primärpräventiven Präventionsziel. Es wird argumentiert, dass Primärprävention, die nach wie vor auf die Abstinenz als erstes Präventionsziel abzielt, vor dem Hintergrund der referierten Befunde zur Wirksamkeit von Präventionsmaßnahmen als auch des entwicklungswissenschaftlichen Standes zur Drogenforschung zu kurz greift.

Schlüisselwörter

Prävention · Präventionsziel · Primärprävention · Wirksamkeit
Key words

Prevention · aim of prevention · primary prevention · efficary
Konzepte der Drogen- und Suchtprävention: Ein Blick in die Geschichte

Drogenpräventive Strategien haben in der Bundesrepublik eine gut 30-jährige Geschichte, in der ganz unterschiedliche Paradigmen der Drogenprävention und Auffassungen über Drogen dominierten. Eine interessante Darstellung der historisch variierenden Verständnisse von Drogenpolitik und -prävention hat Hüsgen präsentiert [1]. Bis 1972, so zeigt ein Blick in die Geschichte, wurde die Drogennachfrage strafrechtlich nicht verfolgt. Handlungsleitend war damals die Position, dass nicht die Substanzen oder ihr Konsum, sondern die psychische Situation des Konsumierenden und die psychologische Funktion des Konsums ggf. einen problematischen Substanzmittelkonsum auslösen können. „Selbst bei den schwersten Suchtgiften wie Heroin oder Morphium“, so schrieben Schmidtbauer u. vom Scheid 1971 im Handbuch Rauschdrogen, sei „es nicht die Droge, welche den Betroffenen süchtig macht, sondern der Betroffene nützt die Droge, um (unbewusst) psychische Konflikte zu mildern, Depressionen zu dämpfen, einer belastenden inneren oder äußeren Situation zu entfliehen; erst dadurch wird er süchtig“ [2]. Entsprechend wurden in der staatlichen Prävention weniger die Gefahren von Drogen als vielmehr Entwicklungsprobleme in Pubertät und Adoleszenz thematisiert. Aufklärung und Hilfe zur Emanzipation von widrigen Lebensumständen und Biografieverläufen standen im Mittelpunkt.

Angesichts des weit verbreiteten Substanzmittelkonsums unter Schülern und Studenten in der sog. Flower-Power-Bewegung entschloss sich der Bundesgesetzgeber in den 70er-Jahren, auch strafrechtlich gegen den Konsum und Handel mit Drogen vorzugehen. Das Betäubungsmittelgesetz wurde am 10.1.1972 erlassen. Es war geleitet von einer neuen, nämlich kriminologisch-ethischen Sichtweise der Drogenprobleme, wonach die Substanzen selbst - losgelöst vom individuellen oder sozialen Kontext, in dem sie genommen werden - das eigentliche Problem darstellen. Entsprechend galt es, die Verfügbarkeit und den Konsum von psychotropen Substanzen einzuschränken und unter Strafe zu stellen. Die Flower-Power-Bewegung - ideologische Basis massenhafter Drogenerfahrungen von Jugendlichen und jungen Erwachsenen - ebbte ab und mit ihr die Prävalenz des Substanzmittelkonsums [1]. Die Arbeitsgesellschaft attribuierte die Veränderungen der Konsumprävalenz auf die vermeintliche Wirksamkeit der repressiven staatlichen Strategie, die Abstinenz zum starren Ziel aller Suchtangebote machte.
Erst die negativen Erfahrungen, vor allem der Anstieg der Zahl der jährlichen Drogentoten, führten in den 80er-Jahren zu einer Neuordnung des Betäubungsmittelrechts und zu einer Abkehr von der strikten Strafandrohung. „Therapie statt Strafe“ war Ausdruck eines Umdenkens von der Drogenpönalisierung zur Suchtvorbeugung. ${ }^{1}$ Dieses Motto leitete eine erste Hinwendung zu lebenskompetenzorientierten Präventionsprogrammen ein, über die eine Normalisierung des Umgangs mit Substanzen erreicht werden sollte. Drogenkonsum wurde - nun einem psychosozialen Modell folgend - als zielgerichtetes Verhalten interpretiert, das dazu dient, die psychische Verfassung zu verändern bzw. psychische Stabilisierung zu erreichen.

Mit dem Aufkommen von HIV und AIDS kamen Mitte der 80erJahre neue gesundheitspolitische Herausforderungen hinzu. Gegen heftigen Widerstand der Vertreter der kriminologisch-ethischen und der medizinischen Sichtweise der Drogenproblematik (bei der ebenfalls die Substanz mit ihrer zugeschriebenen schädigenden Wirkung im Vordergrund steht) wurden schadensminimierende Strategien durchgesetzt, die eine erweiterte Hilfeopportunität für Drogenabhängige (Hilfe vor Strafe) forderten, die zwar nicht zum Ausstieg bereit waren, aber Gesundheitshilfen bedurften. Zugleich sollte verhindert werden, dass sie das HI-Virus an andere übertragen. Einer gewissen Liberalisierung im Konsumentenbereich wurde jedoch gleichzeitig verstärkte Repression gegen den Handel mit Drogen gegenübergestellt. In der drogenpräventiven Praxis entstand so eine Art Patchwork-Konzept, das teils einer kriminologisch-ethischen, einer psychosozialen und einer medizinischen Deutung des Drogenproblems verpflichtet war.

Die in den 90er-Jahren aufkommende zweite Jugendkultur, die mit Drogen assoziiert war (Techno und Rave), führte - vermutlich weil sie nicht mit einer gesellschaftlichen Antihaltung einherging - zu anderen Reaktionen in der Gesellschaft. Aus den Erfahrungen im HIV-Aids-Bereich wurden schadensminimierende Konzepte übertragen: Risikominimierung, nicht mehr Totalabstinenz, sondern die Einübung von Risikokompetenz sollte einen mündigen Umgang mit Partydrogen ermöglichen [3]. Eine solche Präventionskultur wurde möglich, weil hier - anders als bei Cannabiskonsumenten - eine klare Zielgruppe identifiziert werden konnte,

\footnotetext{
${ }^{1}$ Genau genommen handelt es sich hier nicht um Suchtvorbeugung, sondern um die "Heilung" und Rehabilitation bereits "süchtiger" Drogenkonsumenten.
} 
Tab. 1 Ziele der zukünftigen Suchtpolitik

\section{Die zukünftige Suchtpolitik soll sich an folgenden Oberzielen orientieren:}

1. den Beginn des Konsums verhindern oder hinauszögern;

2. riskante Konsummuster frühzeitig erkennen und reduzieren;

3. das Überleben sichern;

4. eine Abhängigkeit mit allen, nah dem aktuellen Stand der Wissenschaft zur Verfügung stehenden Mitteln behandeln;

5. die Verfügbarkeit illegaler Suchtmittel eindämmen.

Dabei sollen neun Teilziele der Erreichung der allgemeinen Ziele dienen:

1. Förderung des allgemeinen Gesundheitsbewusstseins zur Verhinderung der Entstehung von Abhängigkeit;

2. Veränderung des gesellschaftlichen Klimas zu einem kritischeren Umgang mit legalen und illegalen Drogen;

3. Identifikation von Missbrauchsverhalten zum frühestmöglichen Zeitpunkt und Verbesserung der Früherkennung von neuen psychoaktiven Substanzen und Konsummustern;

4. Sensibilisierung für Gefahren des Mischkonsums;

5. Zielgruppenorientierte Unterstützung besonders gefährdeter Gruppen zur Vermeidung von Abhängigkeit;

6. Reduzierung von Unfällen im Straßenverkehr und am Arbeitsplatz unter dem Einfluss von psychoaktiven Substanzen;

7. Gender Mainstreaming in der Sucht- und Drogenpolitik;

8. Verankerung der interdisziplinären Kooperation

9. Bekämpfung der Betäubungsmittelkriminalität.

die sich selbst artikulierte und schadensminimierende Strategien einforderte. Analoge Präventionskampagnen wie im Ecstasybereich blieben für Cannabiskonsumenten (zunächst) ebenso aus wie ein vergleichbares Präventionskonzept. Für Opiatkonsumenten wurden im Jahr 2000 seitens der rot-grünen Regierung die rechtlichen Voraussetzungen für den Betrieb von Drogenkonsumräumen geschaffen und nach den positiven Erfahrungen in der Schweiz wurde 2002 mit dem Start eines klinischen Modellprojektes zur heroingestützten Behandlung eine neue Drogenperspektive durchgesetzt, in der grundsätzlich anerkannt wird, dass „es nicht die Droge ist, die Abhängigkeit schafft oder auch erhält, sondern die jeweilige Erwartungshaltung der Konsumierenden“ [1].

Die aktuelle Praxis der Drogenprävention schwankt zwischen den verschiedenen Modellvorstellungen. Im Richtungsstreit zwischen der Zielvorstellung einer „suchtmittelfreien Gesellschaft“ und dem Ziel eines kompetenten Umgangs mit Drogen in einer „drogenakzeptierenden Gesellschaft“ entstehen Konzepte und Projekte, die z.T. kontradiktorischen Zielgrößen folgen.

Was die wissenschaftlichen Grundlagen der präventiven Bemühungen anbelangt, dominierten in den Anfängen zunächst Intuition und Aktionismus. Der heute als unzutreffend nachgewiesenen Hoffnung folgend, dass Wissen eine hinreichende Voraussetzung für Verhaltensänderungen sei, standen Informationsvermittlung und Aufklärung über die Risiken des Substanzmittelkonsums im Vordergrund der Prävention. Erst in den 80er-Jahren machten Evaluationsstudien deutlich, dass die Prävalenz des Drogenkonsums durch Wissensvermittlung allein nicht zu beeinflussen ist [4]. Ergänzend wurden nun emotionale und affektive Prozesse und ihre Bedeutung für die Verhaltenssteuerung untersucht. Angstappelle, so zeigte sich, schienen durchaus geeignet, Vermeidungsverhalten (Meidung von Drogen) zu initiieren. Entsprechend wurde unter dieser Prämisse einer affektiv orientierten Präventionsstrategie überwiegend mit drastischen Angstappellen gearbeitet.

Ende der 80er-Jahre wurden unter dem Einfluss salutogenetischer Konzepte sog. Risiko-Ressourcen-Modelle entwickelt, in denen einerseits nach risikoerhöhenden Bedingungen ${ }^{2}$ sowie andererseits nach risikomildernden Resilienz- und Schutzfaktoren des Substanzmittelkonsums ${ }^{3}$ gefragt wurde. Insofern bilden mehrdimensionale Modelle heutzutage die Grundlage moderner Konzepte der Suchtprävention, bei denen entwicklungsorientierte Interventionen einen besonderen Stellenwert haben, die Substanzmittelkonsum als aktives Bewältigungsverhalten konzeptualisieren, mit dem Entwicklungsaufgaben gelöst werden sollen. Die Pubertät und Adoleszenzphase gehen mit spezifischen Lebensaufgaben einher, wie Akzeptanz des Umbaus des eigenen Körpers; Entwicklung einer Schul- und Berufskarriere, Ablösung vom Elternhaus, Hinwendung zu einer Gleichaltrigengruppe, Aufbau einer Beziehung mit erotischer und sexueller Komponente, Entwicklung eines eigenen Wert- und Orientierungssystems u.a.. In dieser Phase können Belastungen und Überforderungen auftreten, die zu psychischen oder körperlichen Beeinträchtigungen führen können und den Gebrauch von legalen und illegalen Substanzen zur Befindensregulation nahe legen [5]. Festzuhalten bleibt dabei, wie in einer methodisch anspruchsvollen Längsschnittstudie überzeugend gezeigt wurde, dass der experimentelle, einer Neugiermotivation entspringende Substanzmittelkonsum die Entwicklung von Jugendlichen fördert und er i.d.R. gerade nicht mit negativen Folgen für Gesundheit und Persönlichkeitsentwicklung einhergeht [6]. Franzkowiak und Schlömer leiten daraus das Ziel ab, „die Überführung von jugendlichem Risikoverhalten in lebenslange Risikokompetenz in den Zielkatalog der Suchtprävention aufzunehmen“ [3].

Allgemein haben sich Bund und Länder 2003 in einem „Aktionsplan Drogen und Sucht“ darauf verständigt, den Stellenwert der Suchtprävention weiter zu stärken [7] (Tab.1).

\section{Zur Wirksamkeit suchtpräventiver Strategien}

Um den aktuellen Stand der vor allem metaanalytisch ermittelten Wirksamkeitsgrade von Suchtpräventionsmaßnahmen abbilden zu können, wurden die Datenbanken PSYNDEX, PSYCINFO und MEDLINE nach Metaanalysen zur Effektivität von

\footnotetext{
${ }^{2}$ Risikoerhöhende Bedingungen sind z. B. genetische Prädisposition, Sensation Seeking, dissoziales Verhalten, emotionaler Distress, Schüchternheit, Ängstlichkeit/Depression, positive Einstellungen zum Substanzkonsum, früher Erstkonsum, Verfügbarkeit von Substanzen im Haushalt, substanzkonsumierende Modelle in der Familie, inkonsistentes Erziehungsverhalten, negative Kommunikationsmuster, geringe soziale Kompetenz, drogenkonsumierende Peers, Ablehnung durch Peers, niedrige Preise für Substanzen, Arbeitslosigkeit, Armut, geringe Bildung, soziale Marginalisierung.

${ }^{3}$ Unter risikomildernden Faktoren werden z.B. Kohärenzsinn, Optimismus, Hardiness, sozial-emotionale Kompetenzen, Risikokompetenz, positives Sozialverhalten, Problemlöse- und Selbstkontrollfähigkeiten, Perspektivübernahme, aktives Bewältigungsverhalten, stabile emotionale Beziehungen, offenes unterstützendes Erziehungsklima, gute Ausbildung, Modelle positiven Bewältigungsverhaltens, Unterstützung in Autonomie, emotionale Unterstützung, soziale Unterstützung, positive Peerund Freundschaftsbeziehungen, positive Schulerfahrungen gefasst.
} 
Präventionsmaßnahmen im Suchtbereich für den Zeitraum der letzten fünf Jahre durchsucht. Insgesamt wurden 101 Studien identifiziert.

Als Ergebnis der Sichtung der Studien kann Folgendes festgehalten werden:

1. Die identifizierten Evaluationsstudien beziehen eine Vielzahl von (Modell-)Programmen ein, die in den letzten 30 Jahren vor allem in den USA und den westlichen Industrienationen durchgeführt worden sind.

2. Die meisten Studien untersuchen schulische Präventionsprogramme; seltener wurde die Wirksamkeit von massenmedialen Kampagnen oder von Interventionen, die sich an Eltern von Heranwachsenden oder an Multiplikatoren richten, evaluiert. Inhaltlich stehen Maßnahmen zur Alkohol-, Tabakund zur allgemeinen Prävention illegalen Drogenkonsums im Vordergrund; spezifische Präventionsprogramme, die die Senkung der Inzidenz von Cannabis-Erstkonsum oder die Senkung der Prävalenz des aktuellen Cannabiskonsums zum Gegenstand haben, wurden nicht identifiziert. Hinzu kommt, dass substanzspezifisch ausgerichtete Präventionsprogramme offenbar nicht effektiver wirken als solche, die den Substanzmittelkonsum generell zum Gegenstand machen [8].

3. Drogenpräventionsprogramme zielen auf die Erreichung sehr unterschiedlicher Kriterien, wie die Erhöhung des Wissens über Risiken des Substanzmittelkonsums, die Reduktion der Konsumhäufigkeit, die Erhöhung des Einstiegsalters, die Reduktion des Missbrauchs und die Reduktion von möglichen, mit dem Substanzmittelkonsum einhergehenden Problemen ab. Eine klare Zieldefinition und Zielgruppenfestlegung sind eher die Ausnahme.

4. Schulische Prävention: In verschiedenen, methodisch hochwerigen Studien konnte gezeigt werden, dass der Drogenkonsum von Jugendlichen tatsächlich reduziert werden kann [9], auch wenn die Effekte eher moderat sind. Die Mehrzahl der 30 in einem systematischen Review analysierten Studien erwies sich jedoch als nicht effektiv. Wirksam waren vor allem Interventionen, die ein interaktives Vorgehen (z.B. Peer-Gespräche, Gruppendiskussionen) in den Mittelpunkt stellen. Das Modell sozialer Einflussnahme erwies sich als tragfähige Grundlage für die Konzeptualisierung von wirksamen Interventionsmaßnahmen, sofern Einfluss auf Normen, die Intention und das persönliche Commitment bei der Entscheidung, nicht zu konsumieren, als Prädiktoren genommen wurde. Schließlich deuten die Ergebnisse darauf hin, dass die Wirksamkeit von (sekundären) Präventionsprogrammen gesteigert werden kann, wenn gemeindebezogene Interventionen die schulischen Maßnahmen unterstützen, wenn Peers als Multiplikatoren genutzt und wenn Life Skills trainiert werden.

5. Peer-Programme: In einer weiteren Metaanalyse wurden peer- und experten-(erwachsenen-)orientierte Programme vergleichend geprüft [10]. Verschiedene Studien unterstützen zwar die Annahme einer Überlegenheit von Peer-Programmen, der metaanalytische Vergleich erbringt jedoch keine schlüssigen Ergebnisse. Auch wenn Peer-Programme gegenüber expertendominierten Programmen eine gewisse Überlegenheit aufweisen (standardisierter Differenzwert: $\mathrm{d}=0,24$ ), wurden jedoch so große Differenzen zwischen den Studien gefunden, dass die Ergebnislage nicht als homogen beurteilt werden kann. Es hat den Anschein, als sei- en Personenmerkmale der programmverantwortlichen Peers bzw. erwachsenen Experten ursächlich für die erzielten Effekte. Unterstützung erhält die These einer Überlegenheit von Peer-Programmen auch durch eine von Black, Tobler u. Sciacca vorgelegte, 120 Studien einbeziehende Metaanalyse, wonach interaktive angelegte Peer-Programme bei 'middle school students' einem Frontalunterricht durch Lehrer oder Wissenschaftler überlegen sind [11]. Und auch Bangert-Drows [12] zeigte in einer älteren Metaanalyse, dass Peers vor allem bessere Effekte bezüglich drogenbezogener Einstellungen erzielen, wenn sie als 'Instructional Leaders' das Instrument der Gruppendiskussion nutzen. Verhaltensbezogene Effekte wurden vor allem erzielt, wenn Studenten eingesetzt wurden. Rationale Informationsvermittlung ist lediglich überlegen, wenn es um Wissenszuwächse geht [13].

6. Massenkommunikation: Massenmediale Informationsvermittlung gilt innerhalb von konsistent angelegten Präventionskampagnen als zweites wichtiges Standbein neben personalkommunikativen Maßnahmen, sofern davon ausgegangen werden kann, dass sich die Botschaften wechselseitig stützen $[4,9,10,14]$.

7. Auf Familien bezogene Maßnahmen, bei denen Kinder beispielsweise aus der Schule Aufgaben mit nach Hause bekamen, die sie mit ihren Eltern durchsprechen sollten oder in denen erzieherische Fähigkeiten und kommunikative Kompetenzen trainiert wurden, scheinen positive Ergebnisse zu erzielen. Eltern und Geschwister fungieren als Rollenmodelle für die Heranwachsenden. Viel versprechend können auch Maßnahmen sein, die Hochrisikofamilien oder Familien von hoch risikobehafteten Kindern als Zielgruppe auswählen $[15,16]$.

8. Unklar dagegen ist offenbar noch die Ergebnislage bez. der Wirksamkeit von gemeindebezogenen Maßnahmen $[9,10]$. Vieles spricht jedoch dafür, dass Effektivität gegeben sein kann, wenn gemeindeorientiert ein Set von aufeinander bezogenen Maßnahmen miteinander kombiniert wird. Auch Tobler et al. [8] sehen die Wirksamkeit als noch begrenzt an.

9. Strukturelle und verhältnispräventive Strategien, wie Preissteigerungen, Werbeverbote oder gesetzliche Maßnahmen, so zeigen Studien, die auf die Reduktion des Tabakkonsums ausgerichtet sind, gelten als wirksame Methoden zur Reduktion des Konsums [17]. Ob sie jedoch auf illegale Substanzen, insbesondere Cannabis, übertragen werden können, bleibt fraglich. Die von der Bundesministerin für Gesundheit eingesetzte Drogen- und Suchtkommission zur Verbesserung der Suchtprävention nahm hierzu 2002 deutlich Stellung: „Die sozialwissenschaftliche Forschung hat sich in den letzten Jahrzehnten intensiv mit der Lenkungswirkung von und der Verhaltenssteuerung durch Recht beschäftigt. Die Ergebnisse dabei sind insgesamt eher entmutigend. Viele der dem Recht zugeschriebenen Folgen oder Ergebnisse lassen sich nicht oder durch andere als rechtliche Mittel besser (z. B. mit weniger Nebenwirkungen) erreichen. Dies gilt insbesondere für die verhaltenssteuernde Wirkung des Strafrechts, wo spezialund generalpräventive Effekte nur bedingt nachzuweisen sind“ (S. 29). Und weiter heißt es „Repressive Vorschriften sollten regelmäßig daraufhin überprüft werden, ob sie präventive Maßnahmen behindern. Im Zweifel sollte der Grundsatz „Prävention vor Repression“ gelten“ (S. 31) [18]. 
Unser heutiges Wissen über die Wirksamkeit von Suchtpräventionsmaßnahmen wurde ausnahmslos cannabisunspezifisch generiert. Angesichts der Tatsache, dass Cannabiskonsum überwiegend einer Neugiermotivation entstammt und der Bewältigung von Entwicklungsaufgaben dient, sind der primären Prävention des Cannabiskonsums (Verhinderung des Erstkonsums) gewisse Grenzen gesetzt. Ebenso fehlen bis heute - was die Primärprävention Cannabisprävention anbelangt - oftmals klar definierte Interventionsziele und Zielgruppen. In der vom BMGS 2005 vorgelegten Dokumentation der Fachtagung „Jugendkult Cannabis Risiken und Hilfen" wird zu Recht betont, dass eine wirksame Präventionsmaßnahme neben einer fundierten Analyse der Ausgangssituation messbare Ziele der Cannabisprävention zu operationalisieren hat, eine exakte Festlegung der anzusprechenden Zielgruppen vornehmen muss und ein Evidenzbasierung der Präventionsmaßnahmen anstreben sollte [19]. Gemessen an diesem Anspruch hat Cannabisprävention in der Bundesrepublik noch einen weiten Weg vor sich. Die bisher vorliegenden Evaluationsstudien haben nämlich cannabisspezifische Wirkungen i.d.R. nicht einmal untersucht.

Vor diesem Hintergrund ist es zu begrüßen, dass in jüngerer Zeit angesichts steigender Konsumentenzahlen und steigender Zahlen von Therapiewilligen sekundärpräventiv angelegte Maßnahmen initiiert wurden, die auch evaluiert wurden bzw. werden sollen. Eine Zusammenstellung aktueller Präventionsprojekte im deutschsprachigen Suchtbereich wurde von Kalke, Raschke, Kern et al. vorgelegt [20]. Um eine Übersicht über neuere, spezifisch auf Cannabis bezogene Präventionsprojekte zu bekommen, wurde zunächst eine internetbasierte Suche mit den Schlagwörtern Cannabis, Cannabiskonsum, Prävention und Suchtprävention durchgeführt. Im Anschluss wurden die einschlägigen Datenbanken PSYNDEX und PSYCINFO (ab dem Jahrgang 1997) mit oben genannten Schlagwörtern durchsucht. Relevante Studien, Projektdarstellungen, Berichte und Informationsmaterial wurden entweder aus dem Internet geladen oder direkt bei den entsprechenden Institutionen (z.B. Bundeszentrale für gesundheitliche Aufklärung) angefordert. Grundlage für die Analyse politischer Forderungen hinsichtlich cannabisspezifischer Präventionsangebote sind zum einen die Dokumentation der Fachtagung "Jugendkult Cannabis - Risiken und Hilfen“, die von der Drogenbeauftragten der Bundesregierung im November 2004 durchgeführt wurde [21], zum anderen der Jahresbericht 2004 der Europäischen Beobachtungsstelle für Drogen und Drogensucht (EBDD) [22] sowie der Bericht des nationalen REITOX-Knotenpunkts an die EBDD [23].

\section{Präventionsangebote aus drogenpolitischer Sicht}

\section{Fachtagung zum Thema ,Jugendkult Cannabis - Risiken und Hilfen“"}

Auf einer von der Drogenbeauftragten der Bundesregierung im November letzten Jahres veranstalteten Fachtagung zum Thema 'Jugendkult Cannabis - Risiken und Hilfen' wurden von Expertinnen und Experten aus Politik und Wissenschaft sieben Handlungsempfehlungen zur Prävention und Therapie zusammengestellt [20]. Zwei der sieben beschriebenen Handlungsempfehlungen (Nr. 1 und 4) legen keine Handlungen nahe, sondern liefern vielmehr eine Beschreibung epidemiologischer Daten und mit dem Konsum von Cannabis in Verbindung gebrachter Folgen.
Somit lassen sich folgende fünf Handlungsempfehlungen kurz zusammenfassen:

- Eine sachliche, glaubwürdige und ideologiefreie Diskussion zum Thema Cannabis wird gefordert. Hierbei soll der Schwerpunkt auf den besonderen Risiken des Cannabiskonsums liegen, die immer noch zu wenig wahrgenommen würden.

- Weiterhin werden deutliche Präventionsbotschaften als wichtig erachtet z. B.

- Häufiger Cannabiskonsum ist nicht harmlos, er kann zu physischen, psychischen und sozialen Beeinträchtigungen führen.

- Jugendliche, die in sehr jungem Alter mit dem Konsum beginnen, tragen ein erhöhtes Risiko.

- Der Probierkonsum von Cannabis führt nicht automatisch zur Abhängigkeit.

- Die Thematisierung hinsichtlich der mit dem Konsum von Cannabis verbundenen Risiken soll im Vordergrund stehen.

- Insbesondere wird darauf hingewiesen, dass Präventions- und Interventionsmaßnahmen riskante Konsummuster und ein frühes Einstiegsalter stärker berücksichtigen sollen. Zielgruppenspezifische Maßnahmen sollen entwickelt und bereits erfolgreiche Strategien zur Prävention wie z.B. das Angebot zur Frühintervention bei erstauffälligen Drogenkonsumenten (FreD) übernommen werden.

- Für die Zielgruppe der Konsumenten mit riskantem Konsummuster soll eine glaubwürdige Kommunikation angeboten werden, die mit den Erfahrungen und Lebensweisen der Jugendlichen in Einklang steht. Bereits bestehende innivative Beratungsbeispiele wie „Quit the Shit" und „Realize it“ sollen weiterentwickelt und evaluiert werden.

- Eine bessere Vernetzung der beteiligten Berufe und Personen (Gesundheitsberufe, Bildungsbereich, Eltern, Suchthilfe u.a.) sowie eine bessere Kooperation zwischen Jugend- und Drogenhilfe werden empfohlen.

Der Schwerpunkt der hier referierten Handlungsempfehlungen liegt auf dem Konsumverhalten der Jugendlichen und ist somit der Verhaltensprävention zuzuordnen. Durch die Vermittlung von Wissen speziell über die Risiken des Cannabiskonsums soll einem Konsum zuvorgekommen werden oder aber der Zeitpunkt des ersten Probierens möglichst lange hinausgezögert werden. Diese Botschaften richten sich an (noch) nicht konsumierende bzw. (noch) nicht riskant konsumierende Jugendliche. Aber auch Risikokonsumenten sollen in zielgruppenspezifischen Maßnahmen berücksichtigt werden.

Die Forderung nach einer verbesserten Vernetzung unterschiedlicher Hilfeeinrichtungen und -angebote dagegen entspricht dem verhältnispräventiven Ansatz.

Erkenntnisse über die psychosozialen Bedingungen der Entstehung von abhängigem Verhalten finden in diesen Empfehlungen keine Entsprechung.

\section{Jahresbericht 2004 der Europäischen Beobachtungsstelle für Drogen und Drogensucht (EBDD)}

Der Jahresbericht der Europäischen Beobachtungsstelle für Drogen und Drogensucht gibt Auskunft über den Stand der Drogenproblematik in der Europäischen Union und in Norwegen. In diesem Bericht werden als explizite Präventionsziele die Reduktion 
der Zahl der Erstkonsumenten sowie das Herauszögern des Erstkonsumalters genannt [22].

In der Präventionsarbeit sind diesem Bericht zufolge kaum Initiativen entwickelt worden, die speziell auf den ansteigenden Cannabiskonsum unter Jugendlichen eingehen, da Prävention selten substanzspezifisch ist. Als zwei Hauptstrategien einiger Mitgliedstaaten werden genannt:

- die Warnung vor den Gefahren des Konsums mit dem Verweis auf das Strafvollzugssystem und

- der Versuch, der sozialen Wahrnehmung des Cannabiskonsums mit Hilfe von Massenkampagnen oder zielgruppenspezifischen Kampagnen in den Medien entgegenzuwirken.

Gemäß der Forderung nach einer neuen Risikodebatte zum Konsum von Cannabis sind die in Deutschland bevorzugten Strategien der ersteren Kategorie zuzuordnen. Die soziale Wahrnehmung des Cannabiskonsums ist hingegen nicht Gegenstand massenmedialer Präventionsbemühungen.

\section{Bericht 2004 des nationalen REITOX-Knotenpunkts an die EBDD [23]}

Entsprechend dem Bericht des nationalen REITOX-Knotenpunktes in Deutschland am Institut für Therapieforschung in München beruhen die Ziele und Schwerpunkte der nationalen Drogenpolitik auf vier Säulen:

- Prävention des Drogenkonsums;

- Beratung und Behandlung von Konsumenten;

- Überlebenshilfe und Schadenshilfen;

- Repression und Reduzierung des Angebots.

Zwei Hauptansätze in der Prävention allgemein werden dabei hervorgehoben:

- substanzungebundene Lebenskompetenzprogramme und

- frühinterventive Maßnahmen.

\section{Cannabisspezifische Präventionsprojekte}

Präventionsmaßnahmen, die explizit auf Cannabis abgestimmt sind, sind eher die Ausnahme. Vielfach kommt der Substanz Cannabis neben anderen illegalen Drogen lediglich der Status einer weiteren Substanz zu, für die keine spezifischen 'Behandlungen' vorgesehen sind. Im Folgenden werden exemplarisch vier cannabisbezogene Präventionsprojekte näher vorgestellt und hinsichtlich ihrer Zielsetzungen und deren Umsetzung analysiert. Es handelt sich dabei um die webbasierten Präventionsangebote 'Realize it' und 'Quit the shit' sowie die Frühintervention bei erstauffälligen Drogenkonsumenten 'FreD' und einen von der Bundeszentrale für gesundheitliche Aufklärung (BZgA) herausgegebenen Informationsleitfaden zum Umgang mit Cannabis in der Schule.

\section{„Realize it" - www.realize-it.org}

Das Projekt „Realize it“ ist ein Angebot für junge Cannabiskonsumenten zwischen 15 und 30 Jahren, die ihren Cannabiskonsum reduzieren bzw. ganz einstellen wollen. Dieses Programm wurde in Zusammenarbeit mit dem Bundesministerium für Gesundheit und Soziales in Deutschland und dem Bundesamt für Gesundheit in der Schweiz entwickelt und wird in beiden Län- dern an jeweils drei Standorten angeboten. Das als Kurzintervention konzipierte Programm ist so aufgebaut, dass die Konsumierenden sich unter der Internetadresse www.realize-it.org informieren, ob ihr Konsum risikobehaftet ist oder nicht. Dies geschieht mittels eines kleinen Tests, der sofort online ausgewertet wird. Wer an der Teilnahme des Programms interessiert ist, wird auf Telefonnummern für die Schweiz und Deutschland verwiesen, so dass die Internetplattform lediglich eine erste Anlaufstelle zur Information bezüglich Cannabiskonsum und seinen Risiken darstellt. Sofern die betroffene Person im Einzugsbereich des Programms wohnt, kann sie einen ersten von insgesamt fünf Terminen in einer Beratungsstelle wahrnehmen. Hier wird zunächst geklärt, welches persönliche Ziel der Teilnehmer oder die Teilnehmerin am Ende des Programms erreichen möchte. Der Programminhalt wird somit auf die Personen maßgeschneidert.

Der festgelegte Zeitrahmen beträgt zehn Wochen und beinhaltet zusätzlich eine Gruppenberatung. An den folgenden Terminen sollen die Umstände des Konsums genauer analysiert werden, um Risikosituationen zu erkennen und Kontrollstrategien zu entwickeln. Ein Begleitbuch zu diesem Programm unterstützt die Teilnehmerinnen und Teilnehmer mit Informationen zu Fragen der Konsumreduktion oder dem Ausstieg. Mittels des darin enthaltenen Konsumtagebuchs wird eine Selbstkontrolle bezüglich des Konsums und der eigenen Fortschritte ermöglicht. Eine Evaluation dieses Angebots liegt bislang nicht vor.

\section{„Quit the Shit" - www.drugcom.de}

Im Rahmen dieses Internetangebotes der Bundeszentrale für gesundheitliche Aufklärung (BZgA) können sich die Jugendlichen unter www.drugcom.de beraten lassen, wie sie ihren Cannabiskonsum reduzieren können. Über 50 Tage soll ein interaktives Tagebuch geführt werden, in dem der Cannabiskonsum dokumentiert wird. Per E-mail wird dieses dann an die Beraterinnen und Berater von drugcom geschickt. Einmal in der Woche erhalten die Jugendlichen ein konstruktives Feedback und individuelle Informationen für eine Veränderung im Konsumverhalten. Die Jugendlichen sollen gezielt in selbstkritischer Reflexion des Drogenkonsums geschult und zu suchtpräventivem Verhalten im Alltag befähigt werden. Die Teilnahme ist anonym und kostenlos und richtet sich insbesondere an junge Cannabiskonsumenten.

Das Ziel dieses Internetangebotes ist die Abstinenz, zumindest aber die Reduzierung des Konsums im Sinne einer Risikominderung. Eine Evaluation dieses Angebots steht derzeit noch aus.

\section{Schule und Cannabis - Regeln, Maßnahmen und Intervention. Leitfaden für Schulen und Lehrpersonen}

Dieser Informationsleitfaden zum Umgang mit Cannabis in der Schule wurde von der Bundeszentrale für gesundheitliche Aufklärung im Auftrag des Bundesministeriums für Gesundheit und Soziale Sicherung herausgegeben [24]. Er stellt für Lehrpersonen eine Handreichung zur Behandlung des Themas Cannabis in der Schule dar. Der Leitfaden ist in vier Teile untergliedert, die Schritt für Schritt mögliche Problematiken zu Cannabis aufgreifen. Im ersten Teil werden Informationen als Ausgangspunkt zusammengefasst. Hier lassen sich Antworten auf allgemeine Fragen finden, die sich im Zusammenhang von Schule und Cannabis stellen. Die Frage „Warum hat Cannabis in der Schule nichts verloren?“ wird 
mit dem Verweis auf die ungünstige Wirkung auf das Leistungsund Sozialverhalten der konsumierenden Person beantwortet. Auch das Aufstellen von Regeln zu Cannabis als bindende Richtlinie für alle Beteiligten wird hier erläutert. Mögliche Interventionen bei Regelbrüchen, Hilfestellungen von außen, gesetzliche Vorschriften zu Cannabis und der Einbezug der Eltern sind weitere Eckpunkte des ersten Teils. Der zweite Teil befasst sich damit, wie gemeinsam(e) Regeln zu Cannabis geschaffen werden können. Ein aus Lehrpersonen, SchulsozialarbeiterIn und Elternvertretung gebildetes Suchtpräventionsteam soll eine gemeinsame Haltung zu Cannabis im schulspezifischen Kontext finden, Regeln zu Cannabis aufstellen und Maßnahmen entwerfen für den Fall, dass diese Regeln gebrochen werden. In Informationsrunden für Lehrpersonen, SchülerInnen und Eltern sollen die Regeln und Maßnahmen dann kommuniziert werden.

Hilfestellung bei der Reaktion auf Regelbrüche bietet der dritte Teil an. Fragen zur Einschätzung der Situation lassen sich mit Hilfe von Eckpunkten wie der Häufigkeit der Vorfälle, der Selbstund Fremdgefährdung, des Alters, der Persönlichkeit und des Entwicklungsstands der betroffenen Person etc. abklären.

Das Ziel dieses Interventionsleitfadens ist die Abstinenz von Cannabis im schulischen Rahmen, wobei auch Klassenfahrten und Schulveranstaltungen mit einbezogen sind. Bei der Erstellung von Regeln wird ausdrücklich dazu aufgefordert, auch Regeln zu Alkohol und anderen Drogen im schulischen Kontext zu schaffen [24]. Die Handreichungen für das Erstellen von Regeln und Maßnahmen und auch die Interventionsvorschläge sind übersichtlich strukturiert. Die Präventionsarbeit wird in erster Linie auf die Schule und die daraus resultierende Leistungssituation konzentriert. Eine generelle Auseinandersetzung mit Drogenkonsum jedweder Art im außerschulischen Alltag der Jugendlichen wird weitestgehend ausgespart. Lediglich bei der Behandlung von Interventionsmaßnahmen wird der Fokus auf konsumauslösende Faktoren innerhalb und außerhalb der Schule gelegt. Auch dieses Angebot wurde unseres Wissens bislang nicht evaluiert.

\section{Frühintervention erstauffälliger Drogenkonsumenten (FreD) [25]}

Das Programm FreD ist eine Interventionsmaßnahme für erstauffällige Drogenkonsumenten, die bereits mit der Polizei aufgrund ihres Drogenkonsums in Konflikt geraten sind. Hierbei wird der Tatbestand, „erwischt“ worden zu sein, genutzt, um die Jugendlichen zu einer Verhaltensänderung mittels eines Trainings zu bringen.

FreD richtet sich an Jugendliche und junge Erwachsene im Alter von 14-21 Jahren, die als KonsumentInnen mit Drogen experimentieren und/oder illegale Drogen missbrauchen oder bereits abhängig sind. Nach einem so genannten Intake-Gespräch, das auf die Motivierung der Jugendlichen abzielt, wird ein mehrgliedriger Kurs angeboten. Dieser Kurs wird auf 2 Tage à $4 \mathrm{~h}$ in einem Abstand von 4-8 Wochen verteilt. Die Teilnehmerzahl pro Kurs wird auf $5-10$ beschränkt.

Die Jugendlichen sollen zu einer konstruktiven Auseinandersetzung bezüglich ihres Umgangs mit Drogen und der damit verbundenen Lebensweise angeregt werden. Ins Gespräch zu kommen wird als immanent wichtig betrachtet.
Im Kursangebot wird spezifisches Wissen über Wirkweisen der verschiedenen Drogen vermittelt. Des Weiteren soll zur Reflexion über den eigenen Umgang mit psychoaktiven Substanzen und die zugrunde liegenden Situationen angeregt werden. Gestärkt werden soll zusätzlich die Fähigkeit, eigenverantwortliche Entscheidungen vor dem Hintergrund der Selbst- und Fremdeinschätzung sowie der persönlichen Risikowahrnehmung zu treffen. Auch die regionalen Hilfemöglichkeiten der Jugend- und Drogenhilfe werden hier vorgestellt.

Ein grundlegendes Ziel dieser Maßnahme ist es, die Entwicklung zu einem missbräuchlichen bzw. abhängigen Drogenkonsum zu verhindern.

Die Evaluation dieses Angebots zeigt, dass 87\% der TeilnehmerInnen das Programm für sehr gut befunden haben und $73 \%$ angaben, das Programm weiterempfehlen zu können. Das Ziel der Frühintervention wurde als erreicht bezeichnet und daraufhin eine Ausweitung des Programms beschlossen. Jugendliche, die zwar auffällig in schulischem Umfeld etc., aber nicht polizeilich auffällig Cannabis konsumieren, wurden bislang nicht erreicht. Die Ausweitung des Programms soll dies nun gewährleisten.

In Tab. 2 sind die hier beschriebenen Präventionsangebote in Form einer Kurzübersicht zusammengestellt.

Festgehalten werden kann, dass die vorgestellten cannabisspezifischen Präventionsangebote ausschließlich sekundärpräventiv angelegt sind. Der immer wieder vorgetragenen Forderung nach primärpräventiven Angeboten und Botschaften zur Verhinderung von Cannabiskonsum bzw. zum Hinauszögern des Erstkonsumalters entsprechen keine expliziten Angebote. Ziel der vorgestellten Präventionsmaßnahmen ist vor allem, risikobelastete jugendliche Konsumenten zur Aufgabe oder zumindest zur Reduktion ihres Konsums zu bewegen. Jugendliche, die im Übergang vom experimentellen, gelegentlichen oder gewohnheitsbedingten unproblematischen $[26,27]$ zum schädlichen Konsum stehen, sind bislang nicht als Zielgruppe definiert.

\section{Diskussion und Schlussfolgerungen}

Angesichts eines sich in verschiedenen Studien abzeichnenden Trends zu möglicherweise ansteigenden Lifetime-Prävalenzraten von Cannabiskonsum (vgl. dazu kritisch [28]), angesichts mehrfach publizierter Hinweise auf einen früheren Konsumeinstieg - nicht nur in der Schweiz (vgl. dazu [29]), sondern auch in der Bundesrepublik - und schließlich angesichts einer massenmedial aufbereiteten Vervielfältigung und Umformung dieser Hinweise zu einer skandalisierenden Berichterstattung über „Die Seuche Cannabis“ (vgl. dazu [30]) wird ausgehend vom Bundesministerium für Gesundheit unter Federführung der Drogenbeauftragten des Bundes eine neue Risikodebatte über Cannabis geführt. In diesem Zusammenhang scheint der Ruf nach verstärkter Prävention, nach Früherkennungsinstrumenten, nach Frühintervention und nach wirksamen Behandlungs- und Therapieangeboten verständlich. 
Tab. 2 Kurzdarstellung cannabisspezifischer Präventionsprojekte

\begin{tabular}{|c|c|c|c|}
\hline Projekt & explizite Ziele & umgesetzte Maßnahmen & Zielgruppe \\
\hline $\begin{array}{l}\text { "Quit the shit" } \\
\text { www.drugcom.de }\end{array}$ & $\begin{array}{l}\text { Abstinenz bzw. Reduktion des Cannabiskon- } \\
\text { sums }\end{array}$ & $\begin{array}{l}\text { - interaktives Tagebuch im Internet } \\
\text { - Schulung in kritischer Selbstrefle- } \\
\text { xion in Bezug auf den Konsum } \\
\text { - Programm ist noch nicht evaluiert }\end{array}$ & $\begin{array}{l}\text { Jugendliche Cannabiskonsumenten mit Reduk- } \\
\text { tionswunsch }\end{array}$ \\
\hline $\begin{array}{l}\text { „Realize it“ } \\
\text { www.realize-it.org }\end{array}$ & $\begin{array}{l}\text { individuell angepasst für Teilnehmerlnnen } \\
\text { mit Hinleitung zum Konsumausstieg bzw. zur } \\
\text { Konsumreduktion }\end{array}$ & $\begin{array}{l}\text { - Online-Test für das persönliche } \\
\text { Konsumrisiko } \\
\text { - persönliche Beratung in Therapie- } \\
\text { einrichtung } \\
\text { - Einzel- und Gruppengespräche } \\
\text { - Programm ist noch nicht evaluiert }\end{array}$ & $\begin{array}{l}\text { Junge (Risiko-)Cannabiskonsumenten mit Re- } \\
\text { duktionswunsch zwischen } 15 \text { und } 30 \text { Jahren }\end{array}$ \\
\hline „Schule und Cannabis“ & $\begin{array}{l}\text { Verhinderung von Cannabiskonsum in der } \\
\text { Schule und in schulischem Umfeld }\end{array}$ & $\begin{array}{l}\text { - Regeln zum Cannabiskonsum in } \\
\text { der Schule } \\
\text { - Maßnahmen bei Regelverstößen } \\
\text { und Interventionsleitfaden }\end{array}$ & Lehrpersonal als Multiplikator für Jugendliche \\
\hline $\begin{array}{l}\text { FreD - Frühintervention } \\
\text { erstauffälliger Drogen- } \\
\text { konsumenten }\end{array}$ & $\begin{array}{l}\text { - Wissensvermittlung über die verschiedenen } \\
\text { Drogen und deren Wirkung } \\
\text { - Reflexion über den eigenen Umgang mit } \\
\text { psychoaktiven Substanzen anregen } \\
\text { - auf Folgen des Drogengebrauchs hinweisen } \\
\text { - Fähigkeit, eigenverantwortliche Entschei- } \\
\text { dungen zu stärken } \\
\text { - regionale Hilfemöglichkeiten bekannt ma- } \\
\text { chen } \\
\text { - Entwicklung zum missbräuchlichen bzw. } \\
\text { abhängigen Drogenkonsum verhindern }\end{array}$ & $\begin{array}{l}\text { - Intake-Gespräch } \\
\text { - mehrgliedriges Kursangebot von } \\
\text { insgesamt } 2 \text { Tagen à } 4 \text { Stunden } \\
\text { (5-10 Teilnehmer) }\end{array}$ & $\begin{array}{l}\text { Jugendliche Drogenkonsumenten, die durch } \\
\text { den Besitz von illegalen Drogen polizeilich auf- } \\
\text { fällig geworden sind }\end{array}$ \\
\hline
\end{tabular}

Ein Blick auf Geschichte und Gegenwart der Cannabisprävention lässt eine einheitliche, schlüssige und in ihren Aussagen konsistente Präventionsbotschaft nur schwer erkennen. In den letzten 30 Jahren haben sich verschiedene, derzeit parallel existierende Perspektiven, Sichtweisen und Präventionskonzepte entwickelt, die in manchen Bereichen miteinander unvereinbare Ziele verfolgen und die jeweils andere Fokusse für die Prävention von Cannabis nahe legen. Dies betrifft z. B. die Frage nach dem Hauptziel der Primärprävention, der Verhinderung der Inzidenz des Substanzmittelkonsums (Verhinderung des Erstkonsums). Während aus medizinischer und kriminologischer Sicht vor allem die Vermeidung des Kontakts mit der Substanz im Vordergrund steht, betonen psychosoziale und soziokulturelle Perspektiven auf die Drogenproblematik, gestützt durch entwicklungswissenschaftliche Befunde, dass nicht jeder Konsum von Cannabisprodukten vermieden werden muss, ja der weit überwiegend transitorische (Probier-)Konsum unschädlich ist und funktional ist zur Bewältigung von Entwicklungsaufgaben. Deren Unterstützung zu fördern wäre die zentrale Aufgabe einer am Gesundheitsförderungsgedanken orientierten Prävention. Dabei geht es um die Förderung von sozialen und personalen Ressourcen, um den Erwerb von Risikokompetenz und um die Gestaltung von gesundheitsförderlichen Lebenswelten.

In einem zweiten Schritt haben wir danach gefragt, ob und was in der Suchtprävention als evidenzbasiert gelten kann. Die Bilanz verschiedener Metaanalysen, die hierzu gesichtet wurden, macht deutlich, dass Suchtprävention durchaus effektiv sein kann, obwohl sie alles in allem bisher nur mäßige bis moderate Effekte aufweist. Sie zeigt allerdings auch, dass spezifische Ansätze zur primären Prävention von Cannabis bisher - auch international - nicht vorliegen. Alle Evidenz zur Wirksamkeit der Suchtprävention wurde bisher cannabisunspezifisch ermittelt.
Insgesamt besteht ein eklatanter Mangel an methodisch anspruchsvollen Evaluationsstudien in diesem Bereich [vgl. 20]. Präventionsprogramme, die explizit auf die Senkung des Erstkonsums von Cannabis ausgerichtet sind oder die Erhöhung des Einstiegsalters als Outcome-Kriterien genutzt haben, wurden nicht identifiziert. In der Praxis muss dies jedoch nicht notwendigerweise ein Nachteil sein. Im vermutlich wichtigsten Setting, in dem Suchtprävention praktiziert wird, der Schule, sind generalpräventive Ansätze von legalen und illegalen Drogen angebracht, da spezifische Programme in der Primärprävention keine Überlegenheit gegenüber unspezifischen aufweisen. Generell liegt brauchbare Evidenz im Wesentlichen für Programme im Setting Schule vor, wenn diese partizipativ und interaktiv angelegt waren und wenn Peers aktiv einbezogen wurden. Als viel versprechend bewährten sich auch auf Familien bezogene Präventionsprogramme. Zudem können die Effekte gesteigert werden, wenn die Programmanwendung massenmedial gestützt wird und wenn strukturelle und verhältnispräventiv wirksame Rahmenbedingungen geschaffen werden. Erstaunlich wenige Aussagen existieren zum Setting Betrieb, das sich im Präventionsdiskurs, der im Public-Health-Kontext geführt wird, in besonderer Weise bewährt hat und das in der Regel als „model of good practice“ für wirksame verhältnisgestützte Prävention gilt [31]. Erstaunlich auch, dass der Präventionsdiskurs im Bereich illegaler Drogen bis dato keinerlei Bezug auf das Kernproblem in Sachen Gesundheit aller Industrienationen nimmt: die sozial ungleiche Verteilung von Mortalität und Morbidität sowie von gesundheitsbezogenen Risikofaktoren. Entsprechend den Analysen von Hüsler und Kollegen [32], die 1082 Jugendliche im Alter von 11 - 20 Jahren zu ihrem Cannabiskonsumverhalten und verschiedenen Lebensbereichen befragten, sind neben dem Konsumverhalten auch die soziale Ausgangslage und Befindensbeeinträchtigungen von prädiktivem Wert, was die Entwicklung eines 
riskanten Konsummusters anbetrifft. Hieraus lassen sich in erster Linie Forderungen nach verhältnispräventiven Maßnahmen ableiten, die dann in einem zweiten Schritt von verhaltenspräventiven Maßnahmen flankiert werden sollen [vgl. auch 20]. Ein vermehrtes Berücksichtigen auch ätiologischer Erkenntnisse über die Entwicklung des Suchtgeschehens in der Planung von Präventionsmaßnahmen wäre wünschenswert.

Der Sachverständigenrat zur Begutachtung der Entwicklung im Gesundheitswesen sieht in seinem 2005 vorgelegten Gutachten die Etablierung primärpräventiver Strategien zur Verminderung sozial bedingter Ungleichheit von Gesundheitschancen als Kernaufgabe. Die Cannabisforschung ist dieser Frage bisher so gut wie nicht nachgegangen. Entsprechend tut sie sich in der Prävention auch schwer, konkrete Zielgruppen und Settings für Cannabisprävention zu benennen.

Eine ideologiefreie, sich an Zielgruppen orientierende und vor allem glaubwürdige Kommunikation, wie sie von Seiten der Politik eingefordert wird, wäre von großer Bedeutung für den Erfolg von Präventionskampagnen, da nur eine stärkere Anpassung an die Lebensweise und Erfahrungen von Jugendlichen es ermöglicht, die Ziele auch tatsächlich zu erreichen [33]. Auch wenn Cannabis zu den illegalen Substanzen gehört, hat sich der Konsum von Cannabis in den letzen Jahren im Selbstverständnis von Jugendlichen mit anderen legalen Drogen nahezu auf eine Stufe gestellt wird und von manchen Autoren bereits als Alltagsdroge bezeichnet [34].

Bezieht man diese Forderung auf die in den vorgestellten Präventionsangeboten vermittelten Ziele, so lässt sich deutlich das Ziel der Abstinenz oder der Reduktion mit anschließender Abstinenz feststellen. Dass es einen nicht problematischen Konsum von Cannabis geben kann, wird explizit ausgeklammert. Hier weist der Präventionsdiskurs - vorsichtig ausgedrückt - bezogen auf Cannabis noch starke Defizite auf. Die Zeit emotionsüberfrachteter Auseinandersetzungen über die Risiken des Cannabiskonsums ist noch lange nicht vorbei und auch staatliches Handeln beraubt sich der eingeforderten Glaubwürdigkeit, wenn es Regeln zur Anwendung bringt, die später vom Bundesgerichtshof oder vom Bundesverfassungsgericht als rechtswidrig verworfen werden müssen. ${ }^{2}$

Zielgruppenspezifische Sekundärprävention sollte auf die Gruppe der Jugendlichen ausgerichtet sein, die entweder ein erhöhtes Risiko für schädlichen Drogengebrauch tragen oder bereits problematische Gebrauchsmuster entwickelt haben [35]. Dazu ist es erforderlich, zuverlässige Instrumente zur Früherkennung zur Verfügung zu haben, die sensitiv zwischen gefährdeten und nicht gefährdeten KonsumentInnen differenzieren ([36], vgl. auch [29]).

Primärprävention, die nach wie vor auf die Abstinenz als erstes Präventionsziel abzielt, greift vor dem Hintergrund der hier zusammengestellten Befunde zur Wirksamkeit von Präventionsstrategien als auch dem entwicklungspsychologischen Stand zur Dro-

\footnotetext{
2 Wie zum Beispiel das Entziehen des Führerscheins infolge des Auffindens von Cannabis bei einer Person unabhängig davon, ob diese Person unter Cannabiseinfluss ein Kraftfahrzeug gefahren hat.
}

genforschung zu kurz. Gleichwohl muss Primärprävention einen zentralen Stellenwert im Kanon der Suchthilfeaktivitäten einnehmen. Doch muss sie sich zunehmend mit der Frage auseinander setzen, was es denn vorzubeugen (prävenire) gilt. Hier ist man sich weitgehend darüber einig, dass es der wie auch immer gefasste problematische Konsum ist, der das eigentliche Übel darstellt. Um konsistente und glaubwürdige Prävention umsetzen zu können, muss demnach auch festgelegt werden, was als unschädlich gelten kann. Eine überzeugende Präventionspolitik sollte davon Kenntnis nehmen und Präventionsstrategien an der gesellschaftlichen Realität orientieren. Getreu dem von der Drogenbeauftragten der Bundesregierung formulierten Ziel der Prävention, 'den gesundheitsschädlichen Konsum von Suchtmitteln von vornherein zu verhindern'.

\section{Literatur}

${ }^{1}$ Hüsgen HA. Drogenpolitik und Drogenprävention in der Entwicklung. Prävention 2003; 26 (1): $13-17$

${ }^{2}$ Schmidtbauer W, v Scheid J. Handbuch der Rauschdrogen. Frankfurt: Fischer, 1971, 2004

${ }^{3}$ Franzkowiak P, Schlömer H. Entwicklung der Suchtprävention in Deutschland: Konzepte und Praxis. Suchttherapie 2003; 4: 175-182

${ }^{4}$ Schmidt B. Suchtprävention in der Bundesrepublik Deutschland. Grundlagen und Konzeption. Köln: Bundeszentrale für gesundheitliche Aufklärung, 2004: Bd 24

${ }^{5}$ Hurrelmann K. Legale und illegale Drogen - Wie kann ihr Missbrauch verhindert werden? Suchtreport 2000; 6: 29-36

${ }^{6}$ Shedler J, Block J. Adolescent drug use and psychological health: A longitudinal inquiry. American Psychologist 1990; 45 (5): 612 - 630

7 Die Drogenbeauftragte der Bundesregierung. Aktionsplan Drogen und Sucht. Berlin/ Bonn: Bundesministerium für Gesundheit und Soziale Sicherung, 2003

8 Tobler NS, Roona MR, Ochshorn P et al. School-based adolescent drug prevention programs: 1998 meta-analysis. Journal of Primary Prevention 2000; 20 (4): 275

${ }^{9}$ Cuijpers P. Effective ingredients of school-based drug prevention programs. A systematic review. Addictive Behavior 2002; 27 (6): $1009-1023$

${ }^{10}$ Cuijpers P. Peer-led and adult-led school drug prevention: a metaanalytic comparison. Journal of Drug Education 2002; 32 (2): 107-19

${ }^{11}$ Black DR, Tobler NS, Sciacca JP. Peer helping/involvement: An efficacious way to meet the challenge of reducing alcohol, tobacco, and other drug use among youth? Journal of School Health 1998; 68 (3): $87-93$

${ }^{12}$ Bangert-Drowns RL. The effects of school-based substance abuse education: A meta-analysis. Journal of Drug Education 1988; 18 (3): 243

${ }^{13}$ Bledsoe KL. Effectiveness of drug prevention programs designed for adolescents of color: A meta-analysis. Univ Microfilms International, 2003

${ }^{14}$ Künzel-Böhmer J, Bühringer G, Janik-Konecny T. Expertise zur Primärprävention des Substanzmittelmissbrauchs. Baden Baden: Nomos, 1993

${ }^{15}$ Werch CE, Pappas DM, Carison JM et al. . Short- and longterm effects of a pilot prevention program to reduce alcohol consumption. Substance Use \& Misuse 1998; 33: $2303-2321$

${ }^{16}$ Miller-Heyl et al. DARE to be You: A family-support, early preventionprogram. Journal of primary prevention 1998; 18: 257-285

17 Jha P, Chaloupka FJ. Macro-social influences: The effects of prices and tobacco-control policies on the demand for tobacco products. British Medical Journal 2000; 321: 358 - 361

18 Die Drogen und Suchtkommission beim Bundesministerium für Gesundheit. Stellungnahme der Drogen und Suchtkommission zur Verbesserung der Suchtprävention. Bonn: Bundesministerium für $\mathrm{Ge}-$ sundheit, 2001

${ }^{19}$ Jordan S. Wirksame Präventionsmaßnahmen von cannabisbezogenen Störungen bei Jugendlichen: Die Drogenbeauftragte der Bundesregierung. Jugendkult: Cannabis - Risiken und Hilfen. Berlin: Bundesministerium für Gesundheit und soziale Sicherung, 2005: 103-107 
${ }^{20}$ Kalke J, Raschke P, Kern W et al. Handbuch der Suchtprävention. Programme, Projekte und Maßnahmen aus Deutschland, Österreich und der Schweiz. Freiburg: Lambertus, 2004

${ }^{21}$ Die Drogenbeauftragte der Bundesregierung. Jugendkult: Cannabis Risiken und Hilfen. Berlin: Bundesministerium für Gesundheit und soziale Sicherung, 2005

22 Europäische Beobachtungsstelle für Drogen und Drogensucht. Jahresbericht 2004. Stand der Drogenproblematik in der Europäischen Union und Norwegen, 2004

${ }^{23}$ Simon R, David-Spickermann M, Hüllinghorst R. Bericht 2004 des nationalen REITOX Knotenpunkts an die EBDD. 2004

${ }^{24}$ Bundeszentrale für gesundheitliche Aufklärung. Cannabis und Schule. Regeln, Maßnahmen, Frühintervention. Leitfaden für Schulen und Lehrpersonen. Köln: Bundeszentrale für gesundheitliche Aufklärung. Verfügbar unter: http://www.bzga.de/?uid=5846d616096ca7a074e0 ecaf0364ddb3\&id=medien\&sid=6\&idx $=1181 \& s u b=1$ [15.07.2005].

25 Görgen W, Hartmann R, Oliva H. Frühintervention bei erstauffälligen Drogenkonsumenten - FreD. Ergebnisse der wissenschaftlichen Begleitung [Internet].Verfügbar unter http://www.bmgs.bund.de/deu/ gra/publikationen/p_forschung.php [15.07.2005].

${ }^{26}$ Kleiber D, Soellner R. Cannabiskonsum: Entwicklungstendenzen, Konsummuster und Risiken. Weinheim: Juventa, 1998

${ }^{27}$ Soellner R. Zur Typologie von Cannabiskonsumenten: Die Drogenbeauftragte der Bundesregierung. Jugendkult: Cannabis - Risiken und Hilfen. Berlin: Bundesministerium für Gesundheit und soziale Sicherung, 2005: 71-84

${ }^{28}$ Kalke S, Verthein U, Stöver H i.d.Bd. Seuche Cannabis? Kritische Bemerkungen zu neueren epidemiologischen Studien Suchttherapie, 2005: 3: $108-115$
${ }^{29}$ Neuenschwander M, Frick U, Gmel G et al. Cannabiskonsum in der Schweiz: Prävalenz und Determinanten für Risikokonsum. Suchttherapie, 2005: 3: 126 - 132

${ }^{30}$ Gaßmann R. Die Canabisdiskussion aus der Perspektive von Prävention und Suchthilfe. Suchttherapie, 2005: 3: $97-101$

${ }^{31}$ Deutscher Bundestag Drucksache 15/5670. Gutachten 2005 des Sachverständigenrates zur Begutachtung der Entwicklung im Gesundheitsweisen [Internet]. Verfügbar unter: http://dip.bundestag.de/btd/ 15/056/1505670.pdf [15.07.2005].

32 Hüsler G, Werlen E, Plancherel B. Der Einfluß psychosozialer Faktoren auf den Cannabiskonsum. Suchtmed 2004; 6 (3): 221 - 235

${ }^{33}$ Schmidt B. Suchtprävention bei konsumierenden Jugendlichen: Sekundärpräventive Ansätze in der geschlechtsbezogenen Drogenarbeit. Weinheim: Juventa, 1998

${ }^{34}$ Freitag M, Hurrelmann K. Illegale Alltagsdrogen: Cannabis, Ecstasy, Speed und LSD im Jugendalter. Weinheim: Juventa, 1999

${ }^{35}$ Schmidt B. Zielgruppenspezifische Sekundärprävention. In: Freitag M, Hurrelmann K (Hrsg). Illegale Alltagsdrogen: Cannabis, Ecstasy, Speed und LSD im Jugendalter. Weinheim: Juventa, 1999

${ }^{36}$ Burkhart G. Cannabisprobleme im europäischen Kontext - Zur Zunahme der Behandlungsnachfrage in Europa: Die Drogenbeauftragte der Bundesregierung. Jugendkult: Cannabis - Risiken und Hilfen. Berlin: Bundesministerium für Gesundheit und soziale Sicherung, 2005: $30-40$ 